\title{
22. GRAIN-SIZE AND CARBON-CARBONATE ANALYSES, LEG 58
}

\author{
Stan M. White, Scripps Institution of Oceanography, La Jolla, California
}

\section{GRAIN-SIZE ANALYSES}

Sand-silt-clay distribution was determined on $10-\mathrm{cm}^{3}$ sediment samples collected at the time the cores were split and described. The results are listed in Table 1. (No analyses were done for Hole 446A.)

The sediment classification used here is that of Shepard (1954), with the sand, silt, and clay boundaries based on the Wentworth (1922) scale (Figure 1). Thus the sand, silt, and clay fractions are composed of particles whose diameters are 2000 to $62.5 \mu \mathrm{m}, 62.5$ to 3.91 $\mu \mathrm{m}$, and less than $3.91 \mu \mathrm{m}$, respectively. This classification is applied without regard to sediment type and origin; therefore, the sediment names used in this table may differ from those used elsewhere in this volume; e.g., a silt (in this table) composed of nannofossils may be called a nannofossil ooze in a site chapter.

Standard sieve and pipette methods were used to determine the grain-size distribution. The sediment sample was dried and dispersed in a Calgon solution. If a sediment sample failed to disaggregate, it was treated with a sonic probe and, if necessary, hydrogen peroxide. Sediment samples which resisted this treatment were not analyzed.

The sand fraction was removed by wet sieving, using a $63-\mu \mathrm{m}$ sieve, and the silt and clay fractions were analyzed by standard pipette analysis. Sampling depths and times were calculated from equations derived from the Stokes settling-velocity equation (Krumbein and Pettijohn, 1938, pp. 95-96):

$$
\begin{aligned}
& D=V=\frac{2 g r^{2}\left(d_{1}-d_{2}\right)}{9 \eta} \\
& t=\frac{9 D \eta}{2 g r^{2}\left(d_{1}-d_{2}\right)}
\end{aligned}
$$

where

$$
\begin{aligned}
V= & \text { velocity, in } \mathrm{cm} / \mathrm{s} \\
t= & \text { time, in seconds } \\
D= & \text { depth pipette is inserted, in } \mathrm{cm} \\
g= & \text { gravity, in } \mathrm{cm} / \mathrm{s}^{2 *} \\
r= & \text { radius of individual particles, in cm* } \\
d_{1}= & \text { density of solid particles arbitrarily set at } \\
& 2.675 \mathrm{~g} / \mathrm{cc} \\
d_{2}= & \text { absolute density of distilled water at different } \\
& \text { temperatures (Hodgman et al., } 1960, \mathrm{p} .2129) \\
\eta= & \text { viscosity of distilled water in poises at dif- } \\
& \text { ferent temperatures (Hodgman et al., 1960, p. } \\
& 2181 \text { ) }
\end{aligned}
$$

* Five figures were used in calculations to avoid rounding off variations.
The reproducibility of the grain-size analysis has been tested previously (Boyce, 1972), and it was found that over a period of time, with several operators, the reproducibility for the sand-silt-clay fractions is \pm 2.5 per cent (absolute). For detailed step-by-step procedures, see Volume 4 of the Initial Reports of the Deep Sea Drilling Project.

\section{CARBON-CARBONATE ANALYSES}

Sediments recovered during Leg 58 were analyzed for total carbon and acid-insoluble (organic) carbon, using the LECO WR-12 analyzer, according to the standard technique outlined below.

The $3-\mathrm{cm}^{3}$ sediment samples were first dried and ground into a homogeneous powder. The ground sediment was redried at 105 to $110^{\circ} \mathrm{C}$, and two samples, one $0.1 \mathrm{~g}$, and the other $0.5 \mathrm{~g}$, were weighed into LECO clay crucibles. The $0.5 \mathrm{~g}$ sample was acidified with 10 per cent hydrochloric acid and washed with distilled water. The sample was then dried and analyzed for acid-insoluble carbon. The 0.1-g sample was analyzed for total carbon without further treatment. If the sample contained less than 10 per cent $\mathrm{CaCO}_{3}$, an additional 0.5 -g sample was analyzed, for greater accuracy. The calciumcarbonate percentages were calculated as follows: (per cent total C - per cent organic C) $\times 8.33=$ per cent $\mathrm{CaCO}_{3}$. Although other carbonates may be present, all acid-soluble carbon was calculated as calcium carbonate. All results are given in weight per cent (Table 2).

Detailed descriptions of the technique and theory may be found in Bader, Gerard, et al. (1970) and Boyce and Bode (1972).

\section{REFERENCES}

Bader, R. G., Gerard, R. D., et al., 1970. Init. Repts. DSDP, 4: Washington (U. S. Govt. Printing Office).

Boyce, R. E., 1972. Grain size analyses, Leg 9, Deep Sea Drilling Project. In Hays, J. D., et al., Init. Repts. DSDP, 9: Washington (U. S. Govt. Printing Office), p. 799.

Boyce, R. E., and Bode, G. W., 1972. Carbon and carbonate analyses, Leg 9, Deep Sea Drilling Project. In Hays, J. D., et al., Init. Repts. DSDP, 9: Washington (U. S. Govt. Printing Office), p. 747.

Hodgman, C. D., Weast, R. C. Y., and Selby, S. M., 1960. Handbook of Chemistry and Physics: Cleveland (Chemical Rubber Publishing Co.).

Krumbein, W. C., and Pettijohn, F. J., 1938. Manual of sedimentary petrography: New York (Appleton-Century-Crofts).

Shepard, F. P., 1954. Nomenclature based on sand-silt-clay ratios. J. Sediment. Petrol., 24, 151.

Wentworth, C. K., 1922. A scale of grade and class terms for clastic sediments. J. Geol., 30, 377. 
TABLE 1

Grain-Size Analysis, Leg 58

\begin{tabular}{|c|c|c|c|c|c|}
\hline $\begin{array}{c}\text { Sample } \\
\text { (Interval in } \mathrm{cm})\end{array}$ & $\begin{array}{l}\text { Sub- } \\
\text { Bottom } \\
(\mathrm{m})\end{array}$ & $\begin{array}{l}\text { Sand } \\
(\%)\end{array}$ & $\begin{array}{l}\text { Silt } \\
(\%)\end{array}$ & $\begin{array}{l}\text { Clay } \\
(\%)\end{array}$ & Classification \\
\hline $442 A-2-2,98$ & 11.98 & 0.4 & 47.1 & 52.5 & Silty clay \\
\hline $2-4,76$ & 14.76 & 0.0 & 91.2 & 8.7 & Silt \\
\hline $2-6,50$ & 17.50 & 2.9 & 50.5 & 46.7 & Clayey silt \\
\hline $3-2,41$ & 20.91 & 3.8 & 51.2 & 45.1 & Clayey silt \\
\hline $3-4,70$ & 24.20 & 0.3 & 39.4 & 60.2 & Silty clay \\
\hline $3-6,88$ & 27.38 & 1.3 & 51.8 & 47.0 & Clayey silt \\
\hline $4-2,70$ & 30.70 & 0.9 & 46.8 & 52.3 & Silty clay \\
\hline $4-4,82$ & 33.82 & 0.1 & 49.0 & 50.9 & Silty clay \\
\hline $5-2,74$ & 40.24 & 1.2 & 48.5 & 50.3 & Silty clay \\
\hline $5-4,34$ & 42.84 & 1.9 & 41.1 & 57.1 & Silty clay \\
\hline $5-4,40$ & 42.90 & 1.6 & 47.3 & 51.1 & Silty clay \\
\hline $5-6,10$ & 45.60 & 0.8 & 46.2 & 53.0 & Silty clay \\
\hline $6-2,54$ & 49.54 & 0.3 & 44.8 & 54.9 & Silty clay \\
\hline $6-4,70$ & 52.70 & 0.4 & 55.9 & 43.7 & Clayey silt \\
\hline $6-6,15$ & 55.15 & 0.8 & 42.9 & 56.3 & Silty clay \\
\hline $7-2,60$ & 59.10 & 0.1 & 43.5 & 56.4 & Silty clay \\
\hline $7-4,60$ & 62.10 & 1.1 & 42.0 & 56.9 & Silty clay \\
\hline $8-4,26$ & 71.26 & 0.3 & 42.0 & 57.7 & Silty clay \\
\hline $10-2,124$ & 88.24 & 0.4 & 40.0 & 59.6 & Silty clay \\
\hline $10-4,30$ & 90.30 & 15.8 & 60.2 & 24.0 & Clayey silt \\
\hline $13-2,130$ & 116.80 & 0.5 & 39.4 & 60.1 & Silty clay \\
\hline $14-3,45$ & 126.95 & 0.2 & 39.0 & 60.9 & Silty clay \\
\hline $15-2,11$ & 134.61 & 0.4 & 36.8 & 62.8 & Silty clay \\
\hline $15-4,24$ & 137.74 & 0.1 & 34.7 & 65.2 & Silty clay \\
\hline $16-2,61$ & 144.61 & 0.2 & 32.2 & 67.6 & Silty clay \\
\hline $17-2,51$ & 154.01 & 0.4 & 36.3 & 63.3 & Silty clay \\
\hline $18-2,120$ & 164.20 & 0.5 & 38.1 & 61.5 & Silty clay \\
\hline $19-2,20$ & 172.70 & 1.9 & 35.1 & 62.9 & Silty clay \\
\hline $21-2,43$ & 191.93 & 0.4 & 29.7 & 69.9 & Silty clay \\
\hline $22-1,145$ & 200.95 & 0.6 & 26.9 & 72.5 & Silty clay \\
\hline $23-2,36$ & 210.86 & 0.9 & 28.0 & 71.1 & Silty clay \\
\hline $23-4,36$ & 213.86 & 1.1 & 29.9 & 69.0 & Silty clay \\
\hline $24-2,109$ & 221.09 & 4.8 & 33.7 & 61.5 & Silty clay \\
\hline $24-4,109$ & 224.09 & 1.2 & 37.0 & 61.8 & Silty clay \\
\hline $25-2,41$ & 229.91 & 1.5 & 48.3 & 50.2 & Silty clay \\
\hline $25-4,41$ & 232.91 & 1.1 & 42.6 & 56.2 & Silty clay \\
\hline $26-2,94$ & 239.94 & 1.6 & 41.3 & 57.1 & Silty clay \\
\hline $27-2,103$ & 249.53 & 3.6 & 47.0 & 49.4 & Silty clay \\
\hline $28-2,28$ & 258.28 & 0.5 & 47.8 & 51.7 & Silty clay \\
\hline $28-3,129$ & 260.79 & 7.5 & 73.8 & 18.7 & Clayey silt \\
\hline $29-2,104$ & 268.54 & 1.2 & 48.1 & 50.7 & Silty clay \\
\hline $30-2,26$ & 277.26 & 2.4 & 22.4 & 75.2 & Clay \\
\hline $31-1,22$ & 285.22 & 0.5 & 26.1 & 73.3 & Silty clay \\
\hline $442 B-1-2,73$ & 269.73 & 2.4 & 72.2 & 25.4 & Clayey silt \\
\hline $2-2,38$ & 278.88 & 2.4 & 44.6 & 53.0 & Silty clay \\
\hline $443-1-2,8$ & 1.58 & 0.1 & 34.6 & 65.3 & Silty clay \\
\hline $1-4,76$ & 5.26 & 0.2 & 42.3 & 57.5 & Silty clay \\
\hline $2-2,85$ & 9.35 & 0.4 & 40.0 & 59.6 & Silty clay \\
\hline $2-4,67$ & 12.17 & 0.5 & 40.6 & 58.9 & Silty clay \\
\hline $3-2,68$ & 18.68 & 0.5 & 44.0 & 55.5 & Silty clay \\
\hline $3-4,10$ & 21.10 & 0.6 & 62.4 & 37.0 & Clayey silt \\
\hline $4-2,39$ & 27.89 & 0.3 & 45.4 & 54.3 & Silty clay \\
\hline $4-4,3$ & 30.53 & 0.4 & 45.5 & 54.1 & Silty clay \\
\hline $5-2,20$ & 37.20 & 0.6 & 43.1 & 56.4 & Silty clay \\
\hline $5-4,20$ & 40.20 & 0.3 & 42.2 & 57.4 & Silty clay \\
\hline $6-2,22$ & 46.72 & 0.3 & 46.0 & 53.8 & Silty clay \\
\hline $6-4,26$ & 49.76 & 1.4 & 48.5 & 50.1 & Silty clay \\
\hline $7-6,98$ & 62.98 & 1.1 & 45.1 & 53.8 & Silty clay \\
\hline $8-4,66$ & 69.16 & 0.1 & 39.3 & 60.6 & Silty clay \\
\hline $9-1,74$ & 74.24 & 0.2 & 33.2 & 66.6 & Silty clay \\
\hline $9-4,74$ & 78.74 & 0.3 & 32.9 & 66.8 & Silty clay \\
\hline $9-6,74$ & 81.74 & 0.8 & 43.3 & 55.9 & Silty clay \\
\hline $10-2,125$ & 85.75 & 0.7 & 45.7 & 53.6 & Silty clay \\
\hline
\end{tabular}

TABLE 1 - Continued

\begin{tabular}{|c|c|c|c|c|c|}
\hline $\begin{array}{c}\text { Sample } \\
\text { (Interval in } \mathrm{cm} \text { ) }\end{array}$ & $\begin{array}{l}\text { Sub- } \\
\text { Bottom } \\
\text { (m) }\end{array}$ & $\begin{array}{l}\text { Sand } \\
(\%)\end{array}$ & $\begin{array}{l}\text { Silt } \\
(\%)\end{array}$ & $\begin{array}{l}\text { Clay } \\
(\%)\end{array}$ & Classification \\
\hline $443-10-4,57$ & 88.07 & 1.4 & 44.0 & 54.6 & Silty clay \\
\hline $10-6,57$ & 91.07 & 0.1 & 42.0 & 57.9 & Silty clay \\
\hline $11-2,118$ & 95.18 & 0.6 & 41.0 & 58.4 & Silty clay \\
\hline $11-4,118$ & 98.18 & 0.5 & 50.3 & 49.2 & Clayey silt \\
\hline $11-6,60$ & 100.60 & 0.1 & 44.2 & 55.7 & Silty clay \\
\hline $14-4,80$ & 126.30 & 0.4 & 48.0 & 51.6 & Silty clay \\
\hline $15-2,24$ & 132.24 & 0.2 & 35.8 & 64.0 & Silty clay \\
\hline $15-4,24$ & 135.24 & 0.1 & 34.5 & 65.4 & Silty clay \\
\hline $15-7,18$ & 139.68 & 1.9 & 47.4 & 50.8 & Silty clay \\
\hline $17-4,33$ & 154.33 & 0.7 & 45.6 & 53.7 & Silty clay \\
\hline $18-2,46$ & 160.96 & 0.9 & 50.0 & 49.0 & Clayey silt \\
\hline $21-2,31$ & 189.31 & 0.2 & 26.4 & 73.5 & Silty clay \\
\hline $22-2,125$ & 199.75 & 3.2 & 52.0 & 44.8 & Clayey silt \\
\hline $23-2,25$ & 208.25 & 0.7 & 33.4 & 65.9 & Silty clay \\
\hline $23-4,32$ & 211.32 & 9.1 & 43.1 & 47.8 & Silty clay \\
\hline $24-3,36$ & 219.36 & 2.0 & 40.6 & 57.4 & Silty clay \\
\hline $24-4,127$ & 221.77 & 0.5 & 29.9 & 69.6 & Silty clay \\
\hline $24-6,83$ & 224.33 & 0.5 & 32.7 & 66.8 & Silty clay \\
\hline $25-2,60$ & 227.60 & 0.7 & 38.7 & 60.6 & Silty clay \\
\hline $26-2,48$ & 236.98 & 0.1 & 30.1 & 69.7 & Silty clay \\
\hline $27-2,90$ & 246.90 & 0.6 & 31.7 & 67.6 & Silty clay \\
\hline $28-1,120$ & 255.20 & 0.2 & 33.1 & 66.7 & Silty clay \\
\hline $29-2,94$ & 265.94 & 1.2 & 34.8 & 64.1 & Silty clay \\
\hline $29-4,94$ & 268.94 & 0.3 & 35.5 & 64.2 & Silty clay \\
\hline $30-2,86$ & 275.36 & 1.1 & 43.2 & 55.7 & Silty clay \\
\hline $31-2,27$ & 284.27 & 1.5 & 17.7 & 80.8 & Clay \\
\hline $31-4,27$ & 287.27 & 4.3 & 43.9 & 51.9 & Silty clay \\
\hline $32-1,51$ & 292.51 & 2.3 & 48.6 & 49.1 & Silty clay \\
\hline $33-2,64$ & 303.64 & 3.6 & 49.9 & 46.5 & Clayey silt \\
\hline $34-2,21$ & 312.71 & 3.0 & 49.6 & 47.4 & Clayey silt \\
\hline $34-4,21$ & 315.71 & 4.8 & 52.2 & 42.9 & Clayey silt \\
\hline $35-2,35$ & 322.35 & 0.9 & 40.7 & 58.4 & Silty clay \\
\hline $36-2,24$ & 331.74 & 1.0 & 41.4 & 57.5 & Silty clay \\
\hline $36-4,22$ & 334.72 & 0.5 & 44.2 & 55.3 & Silty clay \\
\hline $39-2,22$ & 360.22 & 0.3 & 37.5 & 62.2 & Silty clay \\
\hline $40-2,36$ & 369.86 & 2.5 & 36.6 & 60.9 & Silty clay \\
\hline $42-1,107$ & 388.07 & 0.3 & 42.8 & 56.9 & Silty clay \\
\hline $43-2,65$ & 398.65 & 4.6 & 51.2 & 44.2 & Clayey silt \\
\hline $45-2,20$ & 417.20 & 2.0 & 49.5 & 48.5 & Clayey silt \\
\hline $46-2,19$ & 426.69 & 1.8 & 45.2 & 53.0 & Silty clay \\
\hline $48-1,105$ & 445.05 & 4.3 & 54.5 & 41.2 & Clayey silt \\
\hline $49-2,45$ & 455.45 & 0.2 & 38.9 & 60.9 & Silty clay \\
\hline $444-2-2,55$ & 8.05 & 0.7 & 37.6 & 61.8 & Silty clay \\
\hline $2-4,43$ & 10.93 & 0.8 & 35.2 & 64.0 & Silty clay \\
\hline $3-2,64$ & 17.64 & 0.1 & 35.3 & 64.6 & Silty clay \\
\hline $4-2,27$ & 26.77 & 16.5 & 52.1 & 31.4 & Clayey silt \\
\hline $4-4,44$ & 29.94 & 4.4 & 48.3 & 47.2 & Clayey silt \\
\hline $5-4,50$ & 39.50 & 2.1 & 46.9 & 51.1 & Silty clay \\
\hline $7-1,64$ & 54.14 & 13.4 & 48.1 & 38.4 & Clayey silt \\
\hline $7-3,93$ & 57.43 & 3.6 & 48.9 & 47.5 & Clayey silt \\
\hline $10-2,38$ & 83.88 & 0.8 & 43.4 & 55.8 & Silty clay \\
\hline $444 \mathrm{~A}-1-2,76$ & 84.26 & 4.4 & 52.5 & 43.1 & Clayey silt \\
\hline $1-4,76$ & 87.26 & 0.7 & 52.7 & 46.6 & Clayey silt \\
\hline $1-6,76$ & 90.26 & 2.1 & 44.1 & 53.8 & Silty clay \\
\hline $2-2,85$ & 93.85 & 8.7 & 81.0 & 10.3 & Silt \\
\hline $3-4,80$ & 106.30 & 0.7 & 43.4 & 55.8 & Silty clay \\
\hline $6-2,24$ & 131.24 & 1.0 & 36.2 & 62.7 & Silty clay \\
\hline $6-5,24$ & 135.74 & 3.7 & 40.6 & 55.8 & Silty clay \\
\hline $7-1,33$ & 139.33 & 1.7 & 41.6 & 56.7 & Silty clay \\
\hline $8-1,48$ & 148.98 & 0.4 & 30.6 & 69.0 & Silty clay \\
\hline $9-2,98$ & 160.48 & 14.9 & 56.7 & 28.4 & Clayey silt \\
\hline $9-4,75$ & 163.25 & 0.5 & 42.5 & 57.0 & Silty clay \\
\hline $10-2,57$ & 169.57 & 14.8 & 55.6 & 29.7 & Clayey silt \\
\hline $11-2,49$ & 178.99 & 0.9 & 80.2 & 18.9 & Silt \\
\hline
\end{tabular}


TABLE 1 - Continued

\begin{tabular}{|c|c|c|c|c|c|}
\hline $\begin{array}{c}\text { Sample } \\
\text { (Interval in } \mathrm{cm} \text { ) }\end{array}$ & $\begin{array}{l}\text { Sub- } \\
\text { Bottom } \\
\text { (m) }\end{array}$ & $\begin{array}{l}\text { Sand } \\
(\%)\end{array}$ & $\begin{array}{l}\text { Silt } \\
(\%)\end{array}$ & $\begin{array}{l}\text { Clay } \\
(\%)\end{array}$ & Classification \\
\hline $444 \mathrm{~A}-11-4,21$ & 181.71 & 0.2 & 74.1 & 25.8 & Clayey silt \\
\hline $12-2,71$ & 188.71 & 0.1 & 37.1 & 62.8 & Silty clay \\
\hline $13-2,16$ & 197.66 & 1.6 & 53.1 & 45.3 & Clayey silt \\
\hline $14-2,11$ & 207.11 & 0.6 & 82.6 & 16.8 & Silt \\
\hline $14-4,11$ & 210.11 & 5.2 & 56.2 & 38.6 & Clayey silt \\
\hline $15-2,93$ & 217.43 & 1.0 & 51.1 & 47.9 & Clayey silt \\
\hline $16-2,105$ & 227.05 & 0.4 & 80.2 & 19.4 & Silt \\
\hline $21-2,89$ & 255.39 & 0.9 & 48.5 & 50.6 & Silty clay \\
\hline $445-1-1,46$ & 0.46 & 1.5 & 37.8 & 60.6 & Silty clay \\
\hline $1-3,106$ & 4.06 & 5.5 & 42.4 & 52.1 & Silty clay \\
\hline $2-1,50$ & 9.00 & 13.9 & 50.9 & 35.2 & Clayey silt \\
\hline $2-3,136$ & 12.86 & 0.8 & 22.8 & 76.4 & Clay \\
\hline $3-1,75$ & 18.75 & 1.7 & 35.1 & 63.2 & Silty clay \\
\hline $3-3,81$ & 21.81 & 1.6 & 31.2 & 67.3 & Silty clay \\
\hline $3-5,46$ & 24.46 & 6.4 & 49.9 & 43.7 & Clayey silt \\
\hline $4-1,20$ & 27.70 & 1.2 & 36.2 & 62.6 & Silty clay \\
\hline $4-3,67$ & 31.17 & 2.7 & 36.1 & 61.2 & Silty clay \\
\hline $4-5,56$ & 34.06 & 0.0 & 34.9 & 65.0 & Silty clay \\
\hline $5-1,2$ & 37.02 & 2.7 & 36.0 & 61.2 & Silty clay \\
\hline $5-3,2$ & 40.02 & 9.9 & 31.6 & 58.6 & Silty clay \\
\hline $6-1,25$ & 46.75 & 3.9 & 33.4 & 62.7 & Silty clay \\
\hline $6-3,25$ & 49.75 & 3.6 & 25.3 & 71.1 & Silty clay \\
\hline $6-5,25$ & 52.75 & 5.1 & 31.1 & 63.9 & Silty clay \\
\hline $7-5,60$ & 62.60 & 2.6 & 27.0 & 70.5 & Silty clay \\
\hline $8-1,90$ & 66.40 & 1.0 & 25.9 & 73.1 & Silty clay \\
\hline $8-3,90$ & 69.40 & 0.0 & 29.0 & 70.9 & Silty clay \\
\hline $8-5,90$ & 72.40 & 0.2 & 27.4 & 72.4 & Silty clay \\
\hline $10-1,57$ & 85.07 & 2.8 & 30.9 & 66.3 & Silty clay \\
\hline $10-3,57$ & 88.07 & 3.3 & 31.6 & 65.1 & Silty clay \\
\hline $10-5,57$ & 91.07 & 3.2 & 33.0 & 63.9 & Silty clay \\
\hline $11-2,62$ & 96.12 & 3.6 & 28.7 & 67.7 & Silty clay \\
\hline $11-4,62$ & 99.12 & 4.8 & 31.9 & 63.3 & Silty clay \\
\hline $12-1,54$ & 104.04 & 0.3 & 24.5 & 75.2 & Clay \\
\hline $12-3,54$ & 107.04 & 0.6 & 29.2 & 70.2 & Silty clay \\
\hline $13-1,48$ & 113.48 & 0.0 & 27.2 & 72.8 & Silty clay \\
\hline $13-3,47$ & 116.47 & 0.0 & 31.9 & 68.1 & Silty clay \\
\hline $14-1,78$ & 123.28 & 0.1 & 27.8 & 72.2 & Silty clay \\
\hline $15-1,63$ & 132.63 & 0.6 & 30.0 & 69.4 & Silty clay \\
\hline $16-1,76$ & 142.26 & 0.0 & 28.1 & 71.9 & Silty clay \\
\hline $16-3,76$ & 145.26 & 35.8 & 38.3 & 25.9 & Sand-silt-clay \\
\hline $17-1,70$ & 151.70 & 0.8 & 27.5 & 71.7 & Silty clay \\
\hline $18-1,43$ & 160.93 & 1.9 & 30.3 & 67.9 & Silty clay \\
\hline $18-3,43$ & 163.93 & 0.1 & 31.5 & 68.4 & Silty clay \\
\hline $18-5,43$ & 166.93 & 0.0 & 39.0 & 61.0 & Silty clay \\
\hline $19-1,100$ & 171.00 & 2.1 & 38.2 & 59.8 & Silty clay \\
\hline $19-3,100$ & 174.00 & 0.7 & 31.7 & 67.6 & Silty clay \\
\hline $20-1,24$ & 179.74 & 1.5 & 33.5 & 65.0 & Silty clay \\
\hline $20-3,24$ & 182.74 & 3.9 & 39.4 & 56.7 & Silty clay \\
\hline $21-1,60$ & 189.60 & 1.6 & 33.1 & 65.3 & Silty clay \\
\hline $22-1,39$ & 198.89 & 1.0 & 27.8 & 71.3 & Silty clay \\
\hline $23-1,29$ & 208.29 & 0.9 & 40.0 & 59.2 & Silty clay \\
\hline $23-3,29$ & 211.29 & 0.6 & 28.1 & 71.3 & Silty clay \\
\hline $24-1,39$ & 217.89 & 0.2 & 21.6 & 78.2 & Clay \\
\hline $24-3,38$ & 220.88 & 4.2 & 46.9 & 48.9 & Silty clay \\
\hline $24-5,44$ & 223.94 & 0.9 & 40.3 & 58.9 & Silty clay \\
\hline $25-1,44$ & 227.44 & 0.3 & 29.9 & 69.8 & Silty clay \\
\hline $25-3,44$ & 230.44 & 1.2 & 32.8 & 66.0 & Silty clay \\
\hline $25-5,44$ & 233.44 & 0.4 & 53.9 & 45.7 & Clayey silt \\
\hline $26-1,51$ & 237.01 & 0.2 & 32.9 & 66.8 & Silty clay \\
\hline $26-3,51$ & 240.01 & 0.0 & 73.9 & 26.1 & Clayey silt \\
\hline $26-6,51$ & 244.51 & 0.0 & 45.2 & 54.8 & Silty clay \\
\hline $27-1,120$ & 247.20 & 1.2 & 34.6 & 64.2 & Silty clay \\
\hline $27-3,120$ & 250.20 & 0.1 & 30.7 & 69.2 & Silty clay \\
\hline $28-1,114$ & 256.64 & 0.1 & 28.0 & 71.8 & Silty clay \\
\hline $29-3,52$ & 268.52 & 0.5 & 33.3 & 66.2 & Silty clay \\
\hline
\end{tabular}

TABLE 1 - Continued

\begin{tabular}{|c|c|c|c|c|c|}
\hline $\begin{array}{l}\text { Sample } \\
\text { (Interval in } \mathrm{cm})\end{array}$ & $\begin{array}{l}\text { Sub- } \\
\text { Bottom } \\
\text { (m) }\end{array}$ & $\begin{array}{c}\text { Sand } \\
(\%)\end{array}$ & $\begin{array}{l}\text { Silt } \\
(\%)\end{array}$ & $\begin{array}{c}\text { Clay } \\
(\%)\end{array}$ & Classification \\
\hline $446-1-1,91$ & 0.91 & 0.2 & 30.9 & 68.9 & Silty clay \\
\hline $3-1,55$ & 11.55 & 0.1 & 29.4 & 70.4 & Silty clay \\
\hline $3-3,55$ & 14.55 & 0.1 & 27.4 & 72.5 & Silty clay \\
\hline $3-5,55$ & 17.55 & 0.1 & 22.2 & 77.7 & Clay \\
\hline $5-3,82$ & 33.82 & 0.6 & 23.4 & 76.0 & Clay \\
\hline $5-5,82$ & 36.82 & 0.3 & 22.0 & 77.7 & Clay \\
\hline $6-1,90$ & 40.40 & 0.1 & 12.7 & 87.1 & Clay \\
\hline $6-3,90$ & 43.40 & 0.1 & 13.5 & 86.4 & Clay \\
\hline $7-1,91$ & 49.91 & 0.0 & 11.4 & 88.6 & Clay \\
\hline $7-4,91$ & 54.41 & 0.1 & 11.7 & 88.2 & Clay \\
\hline $7-5,91$ & 55.91 & 0.1 & 14.2 & 85.7 & Clay \\
\hline $8-1,29$ & 58.79 & 0.1 & 11.0 & 88.9 & Clay \\
\hline $8-4,124$ & 64.24 & 0.1 & 10.9 & 89.0 & Clay \\
\hline $9-1,74$ & 68.74 & 0.8 & 11.5 & 87.7 & Clay \\
\hline $9-3,21$ & 71.21 & 1.5 & 9.9 & 88.6 & Clay \\
\hline $9-5,57$ & 74.57 & 0.0 & 6.4 & 93.5 & Clay \\
\hline $10-1,138$ & 78.88 & 0.1 & 8.7 & 91.2 & Clay \\
\hline $10-3,48$ & 80.98 & 0.1 & 8.9 & 91.0 & Clay \\
\hline $10-5,94$ & 84.44 & 0.1 & 8.6 & 91.3 & Clay \\
\hline $11-1,25$ & 87.25 & 0.0 & 4.4 & 95.6 & Clay \\
\hline $11-1,25$ & 87.25 & 0.0 & 3.4 & 96.6 & Clay \\
\hline $12-1,130$ & 97.80 & 0.1 & 6.8 & 93.2 & Clay \\
\hline $12-3,105$ & 100.55 & 0.1 & 10.8 & 89.1 & Clay \\
\hline $13-1,116$ & 107.16 & 0.0 & 7.2 & 92.7 & Clay \\
\hline $13-3,115$ & 110.15 & 0.0 & 16.4 & 83.5 & Clay \\
\hline $14-1,140$ & 116.90 & 0.0 & 9.5 & 90.5 & Clay \\
\hline $14-3,61$ & 119.11 & 0.0 & 8.4 & 91.6 & Clay \\
\hline $14-5,61$ & 122.11 & 0.0 & 18.1 & 81.9 & Clay \\
\hline $15-1,123$ & 126.23 & 0.2 & 13.9 & 85.9 & Clay \\
\hline $15-3,123$ & 129.23 & 0.2 & 27.0 & 72.8 & Silty clay \\
\hline $16-1,135$ & 135.85 & 0.1 & 16.3 & 83.7 & Clay \\
\hline $16-3,135$ & 138.85 & 0.1 & 21.1 & 78.8 & Clay \\
\hline $18-1,122$ & 154.72 & 0.8 & 32.5 & 66.7 & Silty clay \\
\hline $20-\mathrm{CC}, 10$ & 173.55 & 71.9 & 20.4 & 7.8 & Silty sand \\
\hline $20-\mathrm{CC}, 34$ & 173.55 & 0.5 & 65.7 & 33.8 & Clayey silt \\
\hline $21-1,132$ & 183.32 & 1.5 & 51.6 & 46.9 & Clayey silt \\
\hline $23-1,73$ & 201.73 & 1.1 & 60.7 & 38.2 & Clayey silt \\
\hline $24-1,53$ & 211.03 & 0.9 & 43.8 & 55.3 & Silty clay \\
\hline $24-3,53$ & 214.03 & 0.4 & 65.7 & 33.8 & Clayey silt \\
\hline
\end{tabular}

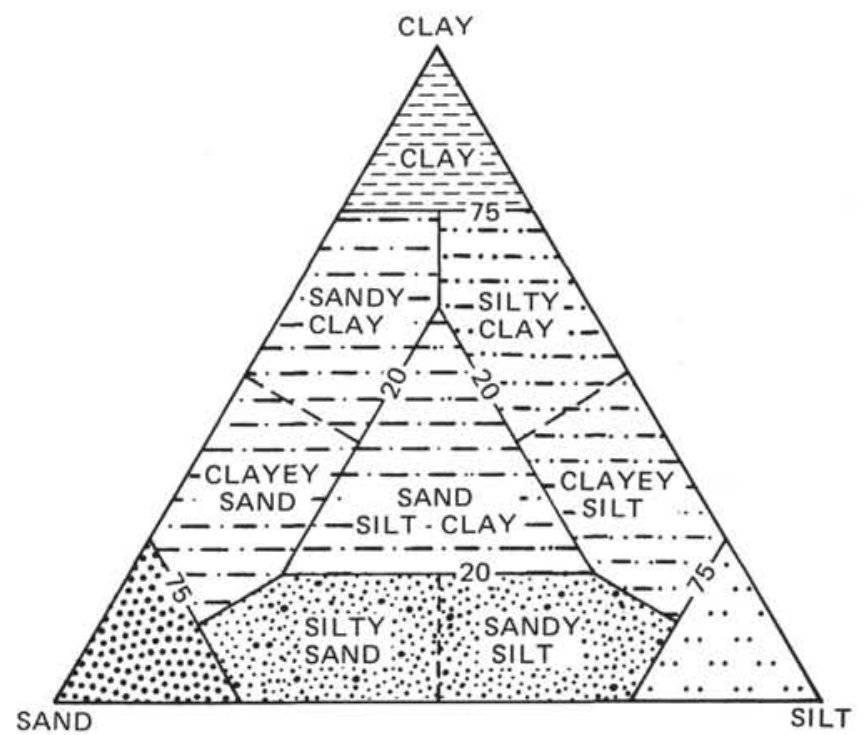

Figure 1. Textural sediment classification (after Shepard, 1954.) 
TABLE 2

Carbon-Carbonate Analyses, Leg 58

\begin{tabular}{|c|c|c|c|c|}
\hline $\begin{array}{c}\text { Sample } \\
\text { (Interval in cm) }\end{array}$ & $\begin{array}{l}\text { Sub-Bottom } \\
\text { Depth } \\
\text { (m) }\end{array}$ & $\begin{array}{l}\text { Total } \\
\text { Carbon } \\
(\%)\end{array}$ & $\begin{array}{c}\text { Organic } \\
\text { Carbon } \\
(\%)\end{array}$ & $\begin{array}{c}\mathrm{CaCO}_{3} \\
(\%)\end{array}$ \\
\hline $442 \mathrm{~A}-3-4,80$ & 24.30 & 0.5 & 0.5 & 0 \\
\hline $3-6,74$ & 27.24 & 0.5 & 0.5 & 0 \\
\hline $4-2,80$ & 30.80 & 0.3 & 0.2 & 1 \\
\hline $4-4,87$ & 33.87 & 0.6 & 0.7 & 0 \\
\hline $5-2,79$ & 40.29 & 0.5 & 0.3 & 2 \\
\hline $5-6,15$ & 45.65 & 0.3 & 0.2 & 1 \\
\hline $6-2,21$ & 49.21 & 0.3 & 0.2 & 1 \\
\hline $6-4,27$ & 52.27 & 0.3 & 0.2 & 1 \\
\hline $6-6,10$ & 55.10 & 0.3 & 0.2 & 1 \\
\hline $7-2,84$ & 59.34 & 0.4 & 0.3 & 1 \\
\hline $7-4,84$ & 62.34 & 0.5 & 0.3 & 2 \\
\hline $8-4,30$ & 71.30 & 0.3 & 0.2 & 0 \\
\hline $10-2,132$ & 88.32 & 0.3 & 0.2 & 1 \\
\hline $10-4,34$ & 90.34 & 0.3 & 0.2 & 1 \\
\hline $13-2,125$ & 116.75 & 0.2 & 0.2 & 0 \\
\hline $14-3,83$ & 127.33 & 0.3 & 0.2 & 1 \\
\hline $15-2,16$ & 134.66 & 0.4 & 0.1 & 2 \\
\hline $15-4,16$ & 137.66 & 0.2 & 0.2 & 0 \\
\hline $16-2,22$ & 144.22 & 0.2 & 0.2 & 0 \\
\hline $17-2,81$ & 154.31 & 0.2 & 0.2 & 0 \\
\hline $18-2,129$ & 164.29 & 0.2 & 0.2 & 1 \\
\hline $19-2,7$ & 172.57 & 0.1 & 0.1 & 0 \\
\hline $21-2,48$ & 191.98 & 0.1 & 0.1 & 0 \\
\hline $23-2,47$ & 210.97 & 0.4 & 0.1 & 3 \\
\hline $23-4 ; 47$ & 213.97 & 0.2 & 0.2 & 0 \\
\hline $24-2,113$ & 221.13 & 0.1 & 0.1 & 0 \\
\hline $24-4,113$ & 224.13 & 0.1 & 0.1 & 0 \\
\hline $25-2,60$ & 230.10 & 0.2 & 0.1 & 1 \\
\hline $25-4,60$ & 233.10 & 0.1 & 0.1 & 0 \\
\hline $26-2,106$ & 240.06 & 0.2 & 0.1 & 1 \\
\hline $27-2,107$ & 249.57 & 0.1 & 0.1 & 0 \\
\hline $28-2,49$ & 258.49 & 0.1 & 0.1 & 0 \\
\hline $29-2,103$ & 268.53 & 0.1 & 0.0 & 0 \\
\hline $30-2,33$ & 277.33 & 0.0 & 0.0 & 0 \\
\hline $442 B-1-2,64$ & 269.64 & 0.1 & 0.1 & 0 \\
\hline $2-2,60$ & 279.10 & 0.1 & 0.1 & 0 \\
\hline $443-1-2,11$ & 1.61 & 0.9 & 0.5 & 3 \\
\hline $1-4,81$ & 5.31 & 0.4 & 0.3 & 1 \\
\hline $2-2,93$ & 9.43 & 0.9 & 0.4 & 5 \\
\hline $2-4,64$ & 12.14 & 1.0 & 0.4 & 5 \\
\hline $3-2,16$ & 18.16 & 0.3 & 0.2 & 1 \\
\hline $3-4,16$ & 21.16 & 0.7 & 0.3 & 3 \\
\hline $3-6,79$ & 24.79 & 0.5 & 0.3 & 1 \\
\hline $4-2,12$ & 27.62 & 1.2 & 0.3 & 8 \\
\hline $4-4,8$ & 30.58 & 0.8 & 0.4 & 3 \\
\hline $5-2,69$ & 37.69 & 0.9 & 0.4 & 5 \\
\hline $5-4,27$ & 40.27 & 0.5 & 0.3 & 2 \\
\hline $6-2,63$ & 47.13 & 1.1 & 0.2 & 7 \\
\hline $6-4,36$ & 49.86 & 0.3 & 0.2 & 1 \\
\hline $7-6,82$ & 62.82 & 1.9 & 0.2 & 15 \\
\hline $8-4,70$ & 69.20 & 0.6 & 0.4 & 2 \\
\hline $9-1,77$ & 74.27 & 1.3 & 0.4 & 7 \\
\hline $9-4,77$ & 78.77 & 1.1 & 0.4 & 6 \\
\hline $9-6,77$ & 81.77 & 0.5 & 0.2 & 2 \\
\hline $10-2,146$ & 85.96 & 0.2 & 0.2 & 0 \\
\hline $10-4,69$ & 88.19 & 0.6 & 0.2 & 3 \\
\hline $10-6,59$ & 91.09 & 0.3 & 0.2 & 1 \\
\hline $11-2,122$ & 95.22 & 1.1 & 0.3 & 7 \\
\hline $11-4,122$ & 98.22 & 0.5 & 0.3 & 2 \\
\hline $11-6,64$ & 100.64 & 0.4 & 0.3 & 1 \\
\hline $14-4,83$ & 126.33 & 0.2 & 0.2 & 0 \\
\hline $15-2,28$ & 132.28 & 0.4 & 0.2 & 1 \\
\hline $15-4,28$ & 135.28 & 0.9 & 0.3 & 5 \\
\hline
\end{tabular}

TABLE 2 - Continued

\begin{tabular}{|c|c|c|c|c|}
\hline $\begin{array}{c}\text { Sample } \\
\text { (Interval in } \mathrm{cm} \text { ) }\end{array}$ & $\begin{array}{l}\text { Sub-Bottom } \\
\text { Depth } \\
\text { (m) }\end{array}$ & $\begin{array}{c}\text { Total } \\
\text { Carbon } \\
(\%)\end{array}$ & $\begin{array}{c}\text { Organic } \\
\text { Carbon } \\
(\%)\end{array}$ & $\begin{array}{c}\mathrm{CaCO}_{3} \\
(\%)\end{array}$ \\
\hline $443-15-7,22$ & 139.72 & 0.2 & 0.2 & 0 \\
\hline $17-4,38$ & 154.38 & 0.5 & 0.2 & 3 \\
\hline $18-2,51$ & 161.01 & 0.3 & 0.2 & 0 \\
\hline $21-2,33$ & 189.33 & 0.3 & 0.2 & 1 \\
\hline $22-2,132$ & 199.82 & 0.8 & 0.1 & 6 \\
\hline $23-2,15$ & 208.15 & 0.3 & 0.1 & 1 \\
\hline $23-4,15$ & 211.15 & 0.2 & 0.1 & 1 \\
\hline $24-3,22$ & 219.22 & 0.7 & 0.1 & 5 \\
\hline $24-4,110$ & 221.60 & 0.2 & 0.1 & 1 \\
\hline $24-6,109$ & 224.59 & 2.0 & 0.1 & 15 \\
\hline $25-2,41$ & 227.41 & 1.0 & 0.1 & 7 \\
\hline $26-2,90$ & 237.40 & 0.5 & 0.1 & 3 \\
\hline $27-2,66$ & 246.66 & 0.3 & 0.1 & 1 \\
\hline $27-4,39$ & 249.39 & 0.2 & 0.1 & 1 \\
\hline $28-1,124$ & 255.24 & 0.1 & 0.1 & 0 \\
\hline $29-2,99$ & 265.99 & 0.4 & 0.1 & 2 \\
\hline $29-4,99$ & 268.99 & 0.2 & 0.1 & 1 \\
\hline $30-2,81$ & 275.31 & 0.2 & 0.1 & 1 \\
\hline $31-2,32$ & 284.32 & 0.2 & 0.2 & 0 \\
\hline $31-4,32$ & 287.32 & 0.2 & 0.1 & 1 \\
\hline $32-1,56$ & 292.56 & 0.3 & 0.1 & 2 \\
\hline $33-2,69$ & 303.69 & 0.6 & 0.1 & 4 \\
\hline $34-2,25$ & 312.75 & 1.6 & 0.1 & 13 \\
\hline $34-4,25$ & 315.75 & 1.0 & 0.1 & 8 \\
\hline $35-2,40$ & 322.40 & 3.3 & 0.1 & 26 \\
\hline $36-2,18$ & 331.68 & 2.3 & 0.1 & 18 \\
\hline $36-4,27$ & 334.77 & 0.9 & 0.1 & 7 \\
\hline $39-2,26$ & 360.26 & 0.6 & 0.1 & 4 \\
\hline $40-2,45$ & 369.95 & 0.9 & 0.1 & 7 \\
\hline $42-2,27$ & 388.77 & 0.5 & 0.1 & 3 \\
\hline $43-2,46$ & 398.46 & 1.1 & 0.1 & 9 \\
\hline $45-2,34$ & 417.34 & 0.1 & 0.1 & 0 \\
\hline $46-2,21$ & 426.71 & 0.1 & 0.1 & 0 \\
\hline $48-1,109$ & 445.09 & 0.8 & 0.4 & 3 \\
\hline $49-2,49$ & 455.49 & 1.5 & 0.0 & 12 \\
\hline $444-2-2,58$ & 8.08 & 0.1 & 0.1 & 0 \\
\hline $2-4,53$ & 11.03 & 0.7 & 0.4 & 3 \\
\hline $3-2,68$ & 17.68 & 0.8 & 0.5 & 3 \\
\hline $4-2,32$ & 26.82 & 0.2 & 0.2 & 1 \\
\hline $4-4,64$ & 30.14 & 0.3 & 0.2 & 1 \\
\hline $5-2,64$ & 36.64 & 0.8 & 0.2 & 5 \\
\hline $5-4,60$ & 39.60 & 0.7 & 0.1 & 4 \\
\hline $7-1,70$ & 54.20 & 0.1 & 0.0 & 0 \\
\hline $7-3,63$ & 57.13 & 0.1 & 0.1 & 0 \\
\hline $7-4,77$ & 58.77 & 0.1 & 0.1 & 0 \\
\hline $10-2,42$ & 83.92 & 0.2 & 0.1 & 1 \\
\hline $444 \mathrm{~A}-1-2,80$ & 84.30 & 1.5 & 0.1 & 11 \\
\hline $1-4,80$ & 87.30 & 0.2 & 0.1 & 1 \\
\hline $1-6,80$ & 90.30 & 0.1 & 0.1 & 1 \\
\hline $2-2,88$ & 93.88 & 0.5 & 0.1 & 4 \\
\hline $3-4,84$ & 106.34 & 0.2 & 0.1 & 1 \\
\hline $6-2,29$ & 131.29 & 0.1 & 0.1 & 0 \\
\hline $6-5,29$ & 135.79 & 0.1 & 0.1 & 0 \\
\hline $7-1,39$ & 139.39 & 0.1 & 0.1 & 0 \\
\hline $8-1,53$ & 149.03 & 0.1 & 0.1 & 0 \\
\hline $9-2,103$ & 160.53 & 0.1 & 0.0 & 0 \\
\hline $9-4,80$ & 163.30 & 2.0 & 0.1 & 16 \\
\hline $10-2,62$ & 169.62 & 0.1 & 0.1 & 0 \\
\hline $11-2,44$ & 178.94 & 0.5 & 0.0 & 4 \\
\hline $11-4,17$ & 181.67 & 1.1 & 0.0 & 9 \\
\hline $12-2,51$ & 188.51 & 3.5 & 0.1 & 29 \\
\hline $13-2,35$ & 197.85 & 0.4 & 0.1 & 3 \\
\hline $14-2,8$ & 207.08 & 0.0 & 0.0 & 0 \\
\hline $14-4,8$ & 210.08 & 0.0 & 0.0 & 0 \\
\hline
\end{tabular}


TABLE 2 - Continued

\begin{tabular}{|c|c|c|c|c|}
\hline $\begin{array}{c}\text { Sample } \\
\text { (Interval in } \mathrm{cm} \text { ) }\end{array}$ & $\begin{array}{l}\text { Sub-Bottom } \\
\text { Depth } \\
\text { (m) }\end{array}$ & $\begin{array}{c}\text { Total } \\
\text { Carbon } \\
(\%)\end{array}$ & $\begin{array}{c}\text { Organic } \\
\text { Carbon } \\
(\%)\end{array}$ & $\begin{array}{c}\mathrm{CaCO}_{3} \\
(\%)\end{array}$ \\
\hline $444 \mathrm{~A}-15-2,91$ & 217.41 & 0.1 & 0.1 & 0 \\
\hline $16-2,108$ & 227.08 & 0.0 & 0.0 & 0 \\
\hline $17-1,21$ & 230.21 & 0.1 & 0.1 & 0 \\
\hline $21-2,90$ & 255.40 & 0.0 & 0.1 & 0 \\
\hline $22-2,25$ & 264.25 & 0.5 & 0.1 & 3 \\
\hline $23-1,31$ & 272.31 & 2.6 & 0.1 & 21 \\
\hline $445-1-1,55$ & 0.55 & 4.7 & 0.3 & 37 \\
\hline $1-3,109$ & 4.09 & 7.8 & 0.1 & 64 \\
\hline $2-1,63$ & 9.13 & 4.8 & 0.2 & 38 \\
\hline $2-3,132$ & 12.82 & 7.2 & 0.1 & 59 \\
\hline $3-1,78$ & 16.78 & 5.1 & 0.1 & 42 \\
\hline $3-3,84$ & 21.84 & 6.4 & 0.1 & 52 \\
\hline $3-5,49$ & 24.49 & 3.2 & 0.1 & 26 \\
\hline 41,23 & 27.73 & 4.1 & 0.1 & 33 \\
\hline $4-3,71$ & 31.21 & 6.4 & 0.1 & 53 \\
\hline $4-5,59$ & 34.09 & 8.2 & 1.0 & 60 \\
\hline $5-1,5$ & 37.05 & 6.8 & 0.1 & 56 \\
\hline $5-3,5$ & 40.05 & 8.2 & 0.7 & 63 \\
\hline $6-1,28$ & 46.78 & 8.4 & 0.9 & 63 \\
\hline $6-3,28$ & 49.78 & 8.4 & 0.1 & 70 \\
\hline $6-5,28$ & 52.78 & 7.2 & 0.1 & 59 \\
\hline $7-5,64$ & 62.64 & 7.8 & 0.1 & 64 \\
\hline $8-1,95$ & 66.45 & 8.0 & 0.1 & 66 \\
\hline $8-3,95$ & 69.45 & 8.1 & 0.1 & 67 \\
\hline $8-5,95$ & 72.45 & 7.9 & 0.1 & 65 \\
\hline $10-1,61$ & 65.11 & 9.2 & 0.1 & 76 \\
\hline $10-3,61$ & 88.11 & 9.1 & 0.1 & 76 \\
\hline $11-2,66$ & 96.16 & 8.6 & 0.1 & 71 \\
\hline $11-4,66$ & 99.16 & 8.1 & 0.1 & 67 \\
\hline $12-1,58$ & 104.08 & 8.4 & 0.1 & 69 \\
\hline $12-3,58$ & 107.08 & 7.9 & 0.1 & 65 \\
\hline $13-1,52$ & 113.52 & 9.2 & 0.1 & 76 \\
\hline $13-3,52$ & 116.52 & 9.0 & 0.1 & 74 \\
\hline $14-1,82$ & 123.32 & 9.7 & 0.1 & 81 \\
\hline $15-1,67$ & 132.67 & 8.1 & 0.1 & 67 \\
\hline $16-1,80$ & 142.30 & 10.1 & 0.0 & 84 \\
\hline $16-3,80$ & 145.30 & 11.0 & 0.1 & 91 \\
\hline $17-1,74$ & 151.74 & 10.1 & 0.1 & 83 \\
\hline $18-1,40$ & 160.90 & 9.7 & 0.1 & 80 \\
\hline $18-3,40$ & 163.90 & 7.3 & 0.1 & 61 \\
\hline $18-5,40$ & 166.90 & 9.3 & 0.0 & 77 \\
\hline $19-1,105$ & 171.05 & 10.2 & 0.0 & 85 \\
\hline $19-3,105$ & 174.05 & 10.3 & 0.0 & 86 \\
\hline $20-1,29$ & 179.79 & 9.5 & 0.0 & 79 \\
\hline $20-3,29$ & 182.79 & 9.5 & 0.0 & 78 \\
\hline $21-1,64$ & 189.64 & 9.1 & 0.0 & 75 \\
\hline $22-1,43$ & 198.93 & 10.5 & 0.5 & 83 \\
\hline $23-1,33$ & 208.33 & 4.2 & 0.1 & 34 \\
\hline $23-3,33$ & 211.33 & 8.9 & 0.1 & 74 \\
\hline $24-1,42$ & 217.92 & 9.4 & 0.1 & 78 \\
\hline $24-3,42$ & 220.92 & 9.1 & 0.0 & 75 \\
\hline $24-5,42$ & 223.92 & 6.8 & 0.1 & 56 \\
\hline $25-1,41$ & 227.41 & 5.1 & 0.1 & 42 \\
\hline $25-3,41$ & 230.41 & 8.9 & 0.1 & 74 \\
\hline $25-5,41$ & 233.41 & 7.3 & 0.1 & 60 \\
\hline $26-1,44$ & 236.94 & 3.7 & 0.1 & 30 \\
\hline $26-3,44$ & 239.94 & 9.5 & 0.1 & 79 \\
\hline $26-6,44$ & 244.44 & 9.3 & 0.0 & 77 \\
\hline $27-1,124$ & 247.24 & 6.8 & 0.1 & 56 \\
\hline $27-3,124$ & 250.24 & 4.9 & 0.1 & 40 \\
\hline $28-1,120$ & 256.70 & 5.5 & 0.1 & 45 \\
\hline $29-2,47$ & 266.97 & 7.0 & 0.1 & 57 \\
\hline $30-1,130$ & 275.80 & 6.7 & 0.1 & 56 \\
\hline $30-3,130$ & 278.80 & 9.2 & 0.0 & 76 \\
\hline $31-1,66$ & 284.66 & 6.9 & 0.0 & 57 \\
\hline
\end{tabular}

TABLE 2 - Continued

\begin{tabular}{|c|c|c|c|c|}
\hline $\begin{array}{c}\text { Sample } \\
\text { (Interval in } \mathrm{cm} \text { ) }\end{array}$ & $\begin{array}{l}\text { Sub-Bottom } \\
\text { Depth } \\
\text { (m) }\end{array}$ & $\begin{array}{l}\text { Total } \\
\text { Carbon } \\
(\%)\end{array}$ & $\begin{array}{l}\text { Organic } \\
\text { Carbon } \\
(\%)\end{array}$ & $\begin{array}{c}\mathrm{CaCO}_{3} \\
(\%)\end{array}$ \\
\hline $445-31-3,66$ & 287.66 & 8.5 & 0.1 & 70 \\
\hline $31-5,66$ & 290.66 & 6.1 & 0.2 & 49 \\
\hline $32-2,76$ & 295.76 & 1.2 & 0.2 & 8 \\
\hline $33-1,89$ & 303.89 & 7.2 & 0.0 & 59 \\
\hline $33-3,89$ & 306.89 & 8.4 & 0.1 & 70 \\
\hline $34-1,140$ & 313.90 & 9.0 & 0.0 & 75 \\
\hline $34-3,140$ & 316.90 & 8.7 & 0.0 & 72 \\
\hline $35-1,88$ & 322.88 & 8.8 & 0.0 & 73 \\
\hline $35-3,88$ & 325.88 & 9.0 & 0.0 & 75 \\
\hline $36-1,39$ & 331.89 & 4.4 & 0.1 & 36 \\
\hline $37-1,90$ & 341.90 & 7.3 & 0.0 & 60 \\
\hline $38-1,77$ & 351.27 & 7.5 & 0.0 & 62 \\
\hline $39-1,82$ & 360.82 & 10.1 & 0.1 & 84 \\
\hline $40-2,63$ & 371.63 & 9.6 & 0.1 & 80 \\
\hline $40-3,79$ & 373.29 & 9.7 & 0.0 & 81 \\
\hline $41-1,44$ & 379.44 & 9.4 & 0.0 & 78 \\
\hline $41-3,44$ & 382.44 & 8.8 & 0.0 & 73 \\
\hline $41-5,44$ & 385.44 & 9.0 & 0.0 & 74 \\
\hline $42-1,23$ & 388.73 & 10.5 & 0.1 & 87 \\
\hline $42-3,30$ & 391.80 & 10.1 & 0.0 & 84 \\
\hline $42-5,38$ & 394.88 & 5.1 & 0.0 & 42 \\
\hline $43-1,85$ & 398.85 & 9.4 & 0.0 & 78 \\
\hline $43-3,85$ & 401.85 & 7.2 & 0.0 & 59 \\
\hline $44-1,121$ & 408.71 & 10.6 & 0.0 & 88 \\
\hline $44-3,121$ & 411.71 & 11.1 & 0.0 & 92 \\
\hline $45-1,124$ & 418.24 & 10.9 & 0.0 & 91 \\
\hline $45-3,124$ & 421.24 & 11.0 & 0.0 & 92 \\
\hline $45-5,39$ & 423.39 & 10.7 & 0.0 & 89 \\
\hline $46-2,98$ & 428.98 & 10.9 & 0.0 & 90 \\
\hline $47-2,44$ & 437.94 & 10.7 & 0.0 & 89 \\
\hline $48-1,114$ & 446.64 & 10.5 & 0.0 & 87 \\
\hline $49-1,110$ & 456.10 & 10.7 & 0.0 & 89 \\
\hline $50-1,36$ & 464.86 & 10.1 & 0.0 & 84 \\
\hline $51-1,33$ & 474.33 & 9.6 & 0.0 & 79 \\
\hline $52-1,22$ & 483.72 & 9.1 & 0.0 & 75 \\
\hline $53-1,70$ & 493.70 & 9.7 & 0.0 & 81 \\
\hline $54-1,61$ & 503.11 & 7.8 & 0.0 & 65 \\
\hline $55-1,74$ & 512.74 & 9.6 & 0.0 & 80 \\
\hline $56-1,66$ & 522.16 & 8.5 & 0.0 & 70 \\
\hline $57-1,93$ & 531.93 & 9.2 & 0.1 & 76 \\
\hline $58-2,55$ & 542.55 & 10.5 & 0.0 & 87 \\
\hline $59-2,17$ & 551.67 & 7.4 & 0.0 & 61 \\
\hline $60-2,67$ & 561.67 & 7.5 & 0.0 & 62 \\
\hline $61-2,99$ & 571.49 & 5.8 & 0.0 & 48 \\
\hline $62-2,61$ & 580.61 & 3.0 & 0.0 & 25 \\
\hline $64-2,137$ & 600.37 & 2.6 & 0.0 & 22 \\
\hline $65-1,50$ & 607.50 & 2.7 & 0.1 & 22 \\
\hline $66-1,60$ & 617.10 & 5.0 & 0.1 & 41 \\
\hline $67-1,70$ & 626.70 & 8.0 & 0.1 & 66 \\
\hline $68-1,17$ & 635.67 & 5.6 & 0.0 & 46 \\
\hline $69-1,77$ & 645.77 & 2.2 & 0.0 & 18 \\
\hline $70-1,62$ & 655.12 & 2.8 & 0.1 & 23 \\
\hline $71-1,124$ & 665.24 & 3.5 & 0.1 & 28 \\
\hline $72-1,21$ & 673.71 & 3.9 & 0.1 & 32 \\
\hline $73-2,94$ & 685.44 & 1.8 & 0.1 & 14 \\
\hline $74-2,89$ & 694.89 & 4.3 & 0.1 & 35 \\
\hline $75-2,90$ & 704.40 & 2.4 & 0.1 & 19 \\
\hline $76-2,74$ & 713.74 & 2.8 & 0.1 & 23 \\
\hline $77-2,23$ & 722.73 & 3.7 & 0.1 & 30 \\
\hline $78-2,18$ & 732.18 & 3.9 & 0.1 & 32 \\
\hline $79-3,46$ & 743.46 & 1.7 & 0.1 & 13 \\
\hline $80-2,59$ & 751.59 & 2.5 & 0.1 & 20 \\
\hline $81-2,41$ & 760.91 & 1.7 & 0.1 & 13 \\
\hline $82-1,20$ & 768.70 & 1.1 & 0.1 & 8 \\
\hline $83-1,104$ & 779.04 & 1.1 & 0.2 & 8 \\
\hline $84-1,120$ & 788.70 & 0.8 & 0.1 & 6 \\
\hline
\end{tabular}


TABLE 2 - Continued

\begin{tabular}{|c|c|c|c|c|}
\hline $\begin{array}{c}\text { Sample } \\
\text { (Interval in cm) }\end{array}$ & $\begin{array}{l}\text { Sub-Bottom } \\
\text { Depth } \\
\text { (m) }\end{array}$ & $\begin{array}{l}\text { Total } \\
\text { Carbon } \\
(\%)\end{array}$ & $\begin{array}{l}\text { Organic } \\
\text { Carbon } \\
(\%)\end{array}$ & $\underset{(\%)}{\mathrm{CaCO}_{3}}$ \\
\hline $445-85-2,64$ & 799.14 & 0.2 & 0.0 & 1 \\
\hline $86-1,66$ & 807.16 & 1.4 & 0.2 & 10 \\
\hline $87-1,70$ & 816.70 & 1.3 & 0.1 & 10 \\
\hline $88-1,94$ & 826.44 & 0.4 & 0.1 & 3 \\
\hline $89-3,57$ & 838.57 & 1.2 & 0.1 & 9 \\
\hline $90-2,108$ & 847.08 & 1.0 & 0.1 & 8 \\
\hline $91-2,56$ & 856.06 & 1.9 & 0.1 & 15 \\
\hline $92-2,45$ & 865.45 & 0.3 & 0.0 & 3 \\
\hline $93-2,140$ & 875.90 & 0.4 & 0.0 & 3 \\
\hline $94-2,102$ & 885.02 & 0.4 & 0.0 & 3 \\
\hline $446 \div 1-1,96$ & 0.96 & 0.2 & 0.2 & 0 \\
\hline $3-1,59$ & 11.59 & 0.1 & 0.1 & 0 \\
\hline $3-3,59$ & 14.59 & 0.1 & 0.1 & 0 \\
\hline $3-5,59$ & 17.59 & 0.1 & 0.1 & 0 \\
\hline $5-3,87$ & 33.87 & 0.1 & 0.1 & 0 \\
\hline $5-5,87$ & 36.87 & 0.1 & 0.1 & 0 \\
\hline $6-3,95$ & 43.45 & 0.1 & 0.1 & 0 \\
\hline $7-1,88$ & 49.88 & 0.1 & 0.1 & 0 \\
\hline $7-5,88$ & 55.88 & 0.1 & 0.1 & 0 \\
\hline $8-1,31$ & 58.81 & 0.1 & 0.1 & 0 \\
\hline $8-4,129$ & 64.29 & 0.1 & 0.1 & 0 \\
\hline $9-1,72$ & 68.72 & 0.1 & 0.1 & 0 \\
\hline $9-3,72$ & 71.72 & 0.1 & 0.1 & 0 \\
\hline $9-5,72$ & 74.72 & 0.1 & 0.1 & 0 \\
\hline $10-1,136$ & 78.86 & 0.1 & 0.1 & 0 \\
\hline $10-3,46$ & 80.96 & 0.0 & 0.0 & 0 \\
\hline
\end{tabular}

TABLE 2 - Continued

\begin{tabular}{|c|c|c|c|c|}
\hline $\begin{array}{c}\text { Sample } \\
\text { (Interval in } \mathrm{cm} \text { ) }\end{array}$ & $\begin{array}{l}\text { Sub-Bottom } \\
\text { Depth } \\
\text { (m) }\end{array}$ & $\begin{array}{c}\text { Total } \\
\text { Carbon } \\
(\%)\end{array}$ & $\begin{array}{c}\text { Organic } \\
\text { Carbon } \\
(\%)\end{array}$ & $\begin{array}{c}\text { Carbon } \\
(\%)\end{array}$ \\
\hline $10-5,92$ & 84.42 & 0.1 & 0.1 & 0 \\
\hline $11-1,29$ & 87.29 & 0.1 & 0.1 & 0 \\
\hline $11-3,29$ & 90.29 & 0.1 & 0.1 & 0 \\
\hline $12-1,32$ & 96.82 & 0.1 & 0.1 & 0 \\
\hline $12-3,32$ & 99.82 & 0.0 & 0.1 & 0 \\
\hline $13-1,98$ & 106.98 & 0.0 & 0.0 & 0 \\
\hline $13-3,99$ & 109.99 & 0.0 & 0.0 & 0 \\
\hline $14-1,101$ & 116.51 & 0.0 & 0.0 & 0 \\
\hline $14-3,101$ & 119.51 & 0.1 & 0.0 & 0 \\
\hline $15-1,127$ & 126.27 & 0.1 & 0.1 & 0 \\
\hline $15-3,127$ & 129.27 & 0.0 & 0.0 & 0 \\
\hline $16-1,140$ & 135.90 & 0.1 & 0.1 & 0 \\
\hline $16-3,140$ & 138.90 & 0.0 & 0.0 & 0 \\
\hline $18-1,126$ & 154.76 & 0.0 & 0.0 & 0 \\
\hline $20-1,6$ & 172.56 & 4.1 & 0.1 & 34 \\
\hline $21-1,136$ & 183.36 & 0.5 & 0.0 & 4 \\
\hline $23-1,77$ & 201.77 & 0.1 & 0.0 & 0 \\
\hline $24-1,52$ & 211.02 & 0.9 & 0.0 & 7 \\
\hline $24-3,52$ & 214.02 & 0.2 & 0.1 & 1 \\
\hline $38-3,128$ & 347.78 & 0.2 & 0.1 & 1 \\
\hline $39-1,31$ & 353.31 & 2.5 & 0.1 & 20 \\
\hline $39-3,31$ & 356.31 & 1.9 & 0.0 & 16 \\
\hline $446 \mathrm{~A}-12-3,85$ & 470.85 & 0.1 & 0.0 & 1 \\
\hline $13-3,64$ & 480.14 & 0.3 & 0.1 & 2 \\
\hline $25-4,14$ & 595.14 & 5.8 & 2.9 & 24 \\
\hline $28-1,32$ & 619.32 & 0.6 & 0.0 & 4 \\
\hline
\end{tabular}

